\title{
On the periodic solutions for some retarded partial differential equations by the use of semi-Fredholm operators
}

\author{
Abdelhai Elazzouzi ${ }^{1}$ \\ KHALIL EzZINBI ${ }^{1,2}$ \\ Mohammed Kriche ${ }^{1}$ \\ 1 Département de Mathématiques, \\ Laboratoire des Sciences de \\ l'Ingénieur (LSI), Faculté \\ Polydisciplinaire de Taza, \\ Université Sidi Mohamed Ben \\ Abdellah (USMBA) - Fes, BP. \\ 1223, Taza, Morocco. \\ abdelhai.elazzouzi@usmba.ac.ma \\ mohammed.kriche@usmba.ac.ma \\ ${ }^{2}$ Département de Mathématiques, \\ Faculté des Sciences Semlalia, \\ Université Cadi Ayyad, B.P. \\ 2390, Marrakesh, Morocco. \\ ezzinbi@uca.ac.ma
}

\begin{abstract}
The main goal of this work is to examine the periodic dynamic behavior of some retarded periodic partial differential equations (PDE). Taking into consideration that the linear part realizes the Hille-Yosida condition, we discuss the Massera's problem to this class of equations. Especially, we use the perturbation theory of semi-Fredholm operators and the Chow and Hale's fixed point theorem to study the relation between the boundedness and the periodicity of solutions for some inhomogeneous linear retarded PDE. An example is also given at the end of this work to show the applicability of our theoretical results.

\section{RESUMEN}

El principal objetivo de este trabajo es examinar el comportamiento dinámico periódico de algunas ecuaciones diferenciales parciales (EDP) periódicas con retardo. Tomando en consideración que la parte lineal cumple la condición de Hille-Yosida, discutimos el problema de Massera para esta clase de ecuaciones. Especialmente usamos la teoría de perturbaciones de operadores semi-Fredholm y el teorema de punto fijo de Chow y Hale para estudiar la relación entre el acotamiento y la periodicidad de soluciones para algunas EDP no homogéneas lineales con retardo. Se entrega un ejemplo al final de este trabajo para mostrar la aplicabilidad de los resultados teóricos.
\end{abstract}

Keywords and Phrases: Hille-Yosida condition, Integral solutions, Semigroup, Semi-Fredholm operators, Periodic solution, Poincaré map.

2020 AMS Mathematics Subject Classification: 34K14, 34K30, 35B10, 35B40. 


\section{Introduction}

Along this work, we investigate the periodicity of solutions of the following inhomogeneous linear retarded PDE

$$
\left\{\begin{aligned}
\frac{d}{d t} y(t) & =\mathcal{A} y(t)+L\left(y_{t}\right)+h(t) & & \text { for } t \geq 0 \\
y(t) & =\psi(t) & & \text { for } \quad-r \leq t \leq 0 .
\end{aligned}\right.
$$

We assume that the generator $\mathcal{A}$ is not necessarily dense on a Banach space $\mathbb{E}$ and realizes the following Hille-Yosida condition:

(i) there exist $\bar{M} \geq 1, \widehat{\omega} \in \mathbb{R}$ such that $(\widehat{\omega}, \infty) \subset \rho(\mathcal{A})$,

(ii) the operator $\mathcal{A}$ satisfies for $n \in \mathbb{N}, \lambda>\widehat{\omega}$, the following inequality

$$
\left|(\lambda I-\mathcal{A})^{-n}\right| \leq \frac{\bar{M}}{(\lambda-\widehat{\omega})^{n}}
$$

where $\rho(\mathcal{A})$ is the resolvent set of $\mathcal{A}$. The history function $y_{t}:[-r, 0] \rightarrow \mathbb{E}$ defined for each $\theta \in[-r, 0]$ by $y_{t}(\theta)=y(t+\theta)$, belongs to $\mathcal{C}([-r, 0], \mathbb{E})$ the space of continuous functions equipped with the supremum norm. $L: \mathcal{C} \rightarrow \mathbb{E}$ is a linear bounded operator and $h$ is a continuous function from $\mathbb{R}$ to $\mathbb{E}$.

Almost periodic and periodic solutions remain the most interesting subject in the qualitative analysis of PDE in view of their important applications in many real phenomena and fields. Recall that the concept of almost periodic is more general than the one of periodicity. It was introduced by Bochner and studied by many authors. For more details on almost periodic function we refer to $[9,16,17,18]$. For the periodicity, there is an extensive literature related to this topic, see for example $[10,11,25]$ for more details. Moreover, the choice of a suitable fixed point theorem is a fundamental tool to establish the periodicity of solutions for different classes of differential equations, in fact, to find a fixed point of the well known Poincaré map is equivalent to find the initial data of the periodic solution of the equation. After a long period of research and development, Massera's theorem [24] is the first result explaining the relation between the existence of bounded and periodic solutions for periodic differential equations. In finite dimensional spaces, several works have been developed on this subject. The authors in $[4,12]$ proved the periodicity of solutions when the solutions of periodic system are just bounded and ultimately bounded by the use of the Horn's fixed point theorem. Especially, in infinite dimensional spaces, the authors in [8], used the Poincaré map approach to get the periodicity of solutions for a class of retarded differential equation, they supposed that the infinitesimal generator satisfies the Hille-Yosida condition and generates a compact semigroup $(\mathcal{T}(t))_{t \geq 0}$ by applying an appropriate fixed point theorem. In [22], the authors proved the periodicity for a nonhomogeneous linear differential equation when the linear part generates a $C_{0}$-semigroup on $\mathbb{E}$ and they obtained the existence and uniqueness of periodic solutions for this class of equations. 
The present work would be a continuation and extension of the work [8] for inhomogeneous linear retarded PDE, we establish the periodicity of solution for Equation (1.1) by using the perturbation theory of semi-Fredholm operators and without considering the compactness condition of $(\mathcal{T}(t))_{t \geq 0}$. To achieve this goal, we suppose that Equation (1.1) admits a bounded solution on the positive real line and under suitable estimations on the norm of the operator $L$, we derive periodic solution of Equation (1.1) from bounded ones on the positive real line by using the perturbation theory of semi-Fredholm operators and the Chow and Hale's fixed point theorem.

This work is treated as follows, in Section 2, we give some definitions and results about integral solutions of Equation (1.1). Moreover, we give some definitions and properties concerning the semiFredholm operators. Section 3 is devoted to prove and introduce some useful estimations on the integral solutions of Equation (1.1). In Section 4, we discuss the problem of existence of periodic solutions of Equation (1.1). Finally, we apply our theoretical results to an equation appearing in physical systems.

\section{Preliminary results}

In this article, we assume that:

$\left(\mathbf{H}_{\mathbf{0}}\right) \mathcal{A}$ satisfies the Hille-Yosida condition.

We consider the following definition and results.

Definition $2.1([1])$. A continuous function $y:[0,+\infty) \rightarrow \mathbb{E}$ is said to be an integral solution of Equation (1.1) if the following conditions hold:

(i) $y:[0,+\infty) \rightarrow \mathbb{E}$ is continuous, such that $y_{0}=\psi$,

(ii) $\int_{0}^{t} y(s) d s \in D(\mathcal{A})$ for $t \geq 0$,

(iii) $y(t)=\psi(0)+\mathcal{A} \int_{0}^{t} y(s) d s+\int_{0}^{t}\left(L\left(y_{s}\right)+h(s)\right) d s$ for $t \geq 0$.

We can deduce from the continuity of the integral solution $y$ that $y(t) \in \overline{D(\mathcal{A})}$, for all $t \geq 0$. Moreover $\psi(0) \in \overline{D(\mathcal{A})}$. In the next we define the part $\mathcal{A}_{0}$ of the operator $\mathcal{A}$ in $\overline{D(\mathcal{A})}$ as follows

$$
D\left(\mathcal{A}_{0}\right)=\{y \in D(\mathcal{A}): \mathcal{A} y \in \overline{D(\mathcal{A})}\}
$$

and

$$
\mathcal{A}_{0} y=\mathcal{A} y \quad \text { for } \quad y \in D\left(\mathcal{A}_{0}\right) .
$$

Lemma $2.2([2])$. The operator $\mathcal{A}_{0}$ is the infinitesimal generator of a strongly continuous semigroup denoted by $\left(\mathcal{T}_{0}(t)\right)_{t \geq 0}$ on $\overline{D(\mathcal{A})}$. 
Theorem $2.3([1])$. Under the assumption $\left(\mathbf{H}_{\mathbf{0}}\right)$. For all $\psi \in \mathcal{C}$ such that $\psi(0) \in \overline{D(\mathcal{A})}$, Equation (1.1) has a unique integral solution $y($.$) on [-r,+\infty)$. Furthermore, $y($.$) is given by$

$$
y(t)=\mathcal{T}_{0}(t) \psi(0)+\lim _{\lambda \rightarrow+\infty} \int_{0}^{t} \mathcal{T}_{0}(t-s) \lambda R(\lambda, \mathcal{A})\left(L\left(y_{s}\right)+h(s)\right) d s \quad \text { for } \quad t \geq 0 .
$$

Through this work, the integral solutions of Equation (1.1) are called plainly solutions. Let $y(., \psi, L, h)$ be the solution of Equation (1.1).

We define $\mathcal{C}_{0}$ the phase space of Equation (1.1) as $\mathcal{C}_{0}=\{\psi \in \mathcal{C}: \psi(0) \in \overline{D(\mathcal{A})}\}$.

Let $\mathcal{X}(t)$ be the linear operator defined on $\mathcal{C}_{0}$ for each $t \geq 0$, by

$$
\mathcal{X}(t) \psi=y_{t}(., \psi, 0,0)
$$

where $y_{t}(., \psi, 0,0)$ is the solution of the following equation

$$
\left\{\begin{aligned}
\frac{d}{d t} y(t) & =A y(t) \quad \text { for } \quad t \geq 0 \\
y_{0} & =\psi
\end{aligned}\right.
$$

Theorem $2.4([1]) \cdot(\mathcal{X}(t))_{t \geq 0}$ is a linear strongly continuous semigroup on $\mathcal{C}_{0}$ :

(i) for all $t \geq 0, \mathcal{X}(t)$ is a bounded linear operator on $\mathcal{C}_{0}$ such that $\mathcal{X}(0)=I$ and $\mathcal{X}(t+s)=$ $\mathcal{X}(t) \mathcal{X}(s)$ for all $t, s \geq 0$,

(ii) for $t \geq 0$ and $\theta \in[-r, 0],(\mathcal{X}(t))_{t \geq 0}$ satisfies:

$$
[\mathcal{X}(t) \phi](\theta)= \begin{cases}{[\mathcal{X}(t+\theta) \phi](0),} & \text { if } \quad t+\theta \geq 0 \\ \phi(t+\theta), & \text { if } t+\theta \leq 0\end{cases}
$$

(iii) for all $\psi \in \mathcal{C}_{0}$ and $t \geq 0, \mathcal{X}(t) \psi$ is a continuous function with values in $\mathcal{C}_{0}$.

Theorem 2.5 ([1]). Under the assumption $\left(\mathbf{H}_{\mathbf{0}}\right)$. The solution $\mathcal{Y}(t) \psi=y_{t}(., \psi, L, 0)$ of Equation (1.1) with $h=0$ can be decomposed as follows:

$$
\mathcal{Y}(t) \psi=\mathcal{X}(t) \psi+\mathcal{Z}(t) \psi \quad \text { for } \quad t \geq 0
$$

where $\mathcal{Z}(t)$, is a bounded linear operator defined on $\mathcal{C}_{0}$, by

$$
[\mathcal{Z}(t) \psi](\theta)=\left\{\begin{array}{cc}
\lim _{\lambda \rightarrow+\infty} \int_{0}^{t+\theta} \mathcal{T}_{0}(t+\theta-s) \lambda R(\lambda, \mathcal{A}) L\left(y_{s}(\psi)\right) d s & t+\theta \geq 0 \\
0 & t+\theta \leq 0
\end{array}\right.
$$

for each $t \geq 0$.

To discuss the existence of periodic solutions, we use the theory of semi-Fredholm operators. We consider some definitions and propositions which are taken from [21]. 
Definition 2.6. Let $\mathbb{E}, \mathbb{F}$ be two Banach spaces. A bounded linear operator $F$ from $\mathbb{E}$ to $\mathbb{F}$, denoted by $F \in \mathcal{L}(\mathbb{E}, \mathbb{F})$, is said to be semi-Fredholm from $\mathbb{E}$ to $\mathbb{F}$ if

(i) $\operatorname{dim} \operatorname{ker}(F)<\infty$, where $\operatorname{ker}(F)$ is the null space.

(ii) $\operatorname{Im}(F)$ the range of $F$ is closed in $\mathbb{F}$.

We designate by $\Phi_{+}(\mathbb{E}, \mathbb{F})$ the set of all semi-Fredholm operators and $\Phi_{+}(\mathbb{E})=\Phi_{+}(\mathbb{E}, \mathbb{E})$. Now we recall some well known theorems for the closed range operators. Let $F \in \mathcal{B}(\mathbb{E}, \mathbb{F})$. Then the quotient space $\mathbb{E} / \operatorname{ker}(F)$ is a Banach space equipped with the norm

$$
|[x]|=\inf \{|x+m|: m \in \operatorname{ker}(F)\},
$$

where

$$
[x]=x+\operatorname{ker}(F):=\{x+m: m \in \operatorname{ker}(F)\} .
$$

Furthermore, if $\operatorname{dim} \operatorname{ker}(F)<\infty$, then there exists a closed subspace $M$ of $\mathbb{E}$ such that

$$
\mathbb{E}=\operatorname{ker}(F) \oplus M
$$

Theorem 2.7 ([21]). Let $F$ be a bounded linear operator in $\mathbb{E}$. Then, $\operatorname{Im}(F)$ is closed if and only if there exists a constant $\widetilde{c}$ such that

$$
|[x]| \leq \widetilde{c}\|F x\| \quad \text { for all } \quad x \in \mathbb{E} .
$$

Theorem 2.8 ([21]). Let $F$ be a bounded linear operator in $\mathbb{E}$ such that $\operatorname{dim} \operatorname{ker}(F)<\infty$. Then, the following assertions are equivalent.

(i) $F \in \Phi_{+}(\mathbb{E})$.

(ii) There exists a constant $\widetilde{c}$ such that

$$
|[x]| \leq \widetilde{c}\|F x\| \quad \text { for all } \quad x \in \mathbb{E} .
$$

(iii) There exists a constant b such that

$$
\|(I-P) x\| \leq b\|F x\| \quad \text { for all } \quad x \in \mathbb{E},
$$

where $P$ is the projection operator onto $\operatorname{ker}(F)$ along $M$.

We present now a result for bounded perturbation of Semi-Fredholm operators.

Theorem $2.9([21])$. Let $F$ be an operator in $\Phi_{+}(\mathbb{E}, \mathbb{F})$. If $S \in \mathbf{L}(\mathbb{E})$ satisfying

$$
\|S\|<\frac{1}{2 b}
$$

where $b$ is the constant given in Theorem 2.8. Then,

$$
F+S \in \Phi_{+}(\mathbb{E}, \mathbb{F}) \quad \text { with } \quad \operatorname{dim} \operatorname{ker}(F+S) \leq \operatorname{dim} \operatorname{ker}(F) .
$$


Now, we need to introduce some well known definitions and results about the spectral theory. Let $\mathcal{J}$ be a linear bounded operator on $\mathbb{F}$, we define the measure of Kuratowski of noncompactness of the operator $\mathcal{J}$ as follows

$$
|\mathcal{J}|_{\alpha}=\inf \{\epsilon>0: \alpha(\mathcal{J}(B)) \leq \epsilon \alpha(B), \text { for every bounded set } B \subset \mathbb{F}\},
$$

where $\alpha($.$) is the measure of Kuratowski of noncompactness of bounded sets B \subset \mathbb{F}$ defined by

$$
\alpha(J)=\inf \{\epsilon>0: B \text { has a finite cover of ball of diameter }<\epsilon\} .
$$

The essential radius $r_{\text {ess }}(\mathcal{J})$ is characterized by the following Nussbaum Formula introduced in [19]:

$$
r_{\text {ess }}(\mathcal{J})=\lim _{n \rightarrow+\infty}\left[\left|\mathcal{J}^{n}\right|_{\alpha}\right]^{1 / n}
$$

Moreover, if $\mathcal{J}$ is bounded and $r_{\text {ess }}(\mathcal{J})<1$, then $I-\mathcal{J} \in \Phi_{+}(\mathbb{F})$.

Let us define the essential growth bound of a strongly continuous semigroup $\mathcal{S}:=(\mathcal{S}(t))_{t \geq 0}$ on a Banach space $\mathbb{F}$ as

$$
\omega_{e s s}(\mathcal{S}):=\lim _{t \rightarrow+\infty} \frac{1}{t} \log |\mathcal{S}(t)|_{\alpha}
$$

It is well know that

$$
r_{\text {ess }}(\mathcal{S}(t))=\exp \left(t \omega_{\text {ess }}(\mathcal{S})\right), \quad t>0
$$

\section{Several estimates}

Before discussing the periodicity of solution of Equation (1.1), we need some preparatory estimates.

Proposition 3.1. Suppose that $\left|\mathcal{T}_{0}(t)\right| \leq M_{0} e^{\omega_{0} t}$ for $t \geq 0$. Then

$$
\|\mathcal{Z}(t)\|_{\mathcal{C}} \leq M_{0} e^{\omega_{0}^{+} t}\left(e^{M_{0}|L| \bar{M} t}-1\right) \text { for } t \geq 0
$$

where $\omega_{0}^{+}=\max \left\{\omega_{0}, 0\right\}$.

To prove the above Proposition, we need to introduce the following Lemma.

Lemma 3.2. Let $\left|\mathcal{T}_{0}(t)\right| \leq M_{0} e^{\omega_{0} t}$ for $t \geq 0$. Then, the solution of Equation (1.1) in the case where $h=0$ is estimated as

$$
|\mathcal{Y}(t)| \leq M_{0} e^{\left(\omega_{0}^{+}+M_{0}|L| \bar{M}\right) t}
$$

Proof. For $t \geq 0, \theta \in[-r, 0]$, one has

$$
\|\mathcal{Y}(t) \psi\|_{\mathcal{C}}=\sup _{\theta \in[-r, 0]}|y(t+\theta, \psi)|=\sup _{\xi \in[t-r, t]}|y(\xi, \psi)| .
$$


Then for $t \geq r$,

$$
\begin{aligned}
\sup _{\xi \in[t-r, t]}|y(\xi, \psi)| & \leq \sup _{\xi \in[t-r, t]}\left|\mathcal{T}_{0}(\xi) \psi(0)\right|+\sup _{\xi \in[t-r, t]}\left|\lim _{\lambda \rightarrow+\infty} \lambda \int_{0}^{\xi} \mathcal{T}_{0}(\xi-s) R(\lambda, \mathcal{A}) L(\mathcal{Y}(s) \psi) d s\right| \\
& \leq M_{0} e^{\omega_{0}^{+} t}\|\psi\|_{\mathcal{C}}+M_{0}|L| \bar{M}\left(\int_{0}^{t} e^{\omega_{0}^{+}(t-s)}\|\mathcal{Y}(s) \psi\|_{\mathcal{C}} d s\right) .
\end{aligned}
$$

For $t<r$,

$$
\begin{aligned}
\sup _{\xi \in[t-r, t]}|y(\xi, \psi)| & =\max \left\{\sup _{\xi \in[t-r, 0]}|y(\xi, \psi)| ; \sup _{\xi \in[0, t]}|y(\xi, \psi)|\right\} \\
& \leq \max \left\{\|\psi\|_{\mathcal{C}} ; \sup _{\xi \in[0, t]}|y(\xi, \psi)|\right\}
\end{aligned}
$$

and

$$
\sup _{\xi \in[0, t]}|y(\xi, \psi)| \leq M_{0} e^{\omega_{0}^{+} t}\|\psi\|_{\mathcal{C}}+M_{0}|L| \bar{M}\left(\int_{0}^{t} e^{\omega_{0}^{+}(t-s)}\|\mathcal{Y}(s) \psi\|_{\mathcal{C}} d s\right) .
$$

Finally, we obtain that

$$
\|\mathcal{Y}(t) \psi\|_{\mathcal{C}} \leq M_{0} e^{\omega_{0}^{+} t}|\psi|_{\mathcal{C}}+M_{0}|L| \bar{M} \int_{0}^{t} e^{\omega_{0}^{+}(t-s)}\|\mathcal{Y}(s) \psi\|_{\mathcal{C}} d s
$$

Hence,

$$
\left\|e^{-\omega_{0}^{+} t} \mathcal{Y}(t) \psi\right\|_{\mathcal{C}} \leq M_{0}\|\psi\|_{\mathcal{C}}+M_{0}|L| \bar{M} \int_{0}^{t} e^{-\omega_{0}^{+} s}\|\mathcal{Y}(s) \psi\|_{\mathcal{C}} d s
$$

Gronwall's inequality implies that

$$
\left\|e^{-\omega_{0}^{+} t} \mathcal{Y}(t) \psi\right\|_{\mathcal{C}} \leq M_{0} e^{M_{0}|L| \bar{M} t}\|\psi\|_{\mathcal{C}}
$$

and then

$$
\|\mathcal{Y}(t) \psi\|_{\mathcal{C}} \leq M_{0} e^{\left(\omega_{0}^{+}+M_{0}|L| \bar{M}\right) t}\|\psi\|_{\mathcal{C}}
$$

Proof of Proposition 3.1. Let $t \geq 0, t+\theta \geq 0$. Then

$$
\begin{aligned}
\|\mathcal{Z}(t) \psi\|_{\mathcal{C}} & =\sup _{\theta \in[-r, 0]}|(\mathcal{Z}(t) \psi)(\theta)| \\
& \leq M_{0}|L| \bar{M}\left(\int_{0}^{t} e^{\omega_{0}^{+}(t-s)}\|\mathcal{Y}(s) \psi\|_{\mathcal{C}} d s\right) .
\end{aligned}
$$

From Lemma 3.2, we have

$$
\begin{aligned}
\|\mathcal{Z}(t) \psi\|_{\mathcal{C}} & \leq M_{0}^{2}|L| \bar{M} e^{\omega_{0}^{+} t}\left(\int_{0}^{t} e^{M_{0}|L| \bar{M} s} d s\right)\|\psi\|_{\mathcal{C}} \\
& \leq M_{0} e^{\omega_{0}^{+} t}\left(e^{M_{0}|L| \bar{M} t}-1\right)\|\psi\|_{\mathcal{C}} .
\end{aligned}
$$

This implies our inequality.

Proposition 3.3. A function $\phi \in \operatorname{ker}(I-\mathcal{X}(\omega))$ if and only if $\phi(0) \in \operatorname{ker}\left(I-\mathcal{T}_{0}(\omega)\right)$, furthermore $\operatorname{dim} \operatorname{ker}(I-\mathcal{X}(\omega))=\operatorname{dim} \operatorname{ker}\left(I-\mathcal{T}_{0}(\omega)\right)$. 
Proof. Let $\phi \in \operatorname{ker}(I-\mathcal{X}(\omega))$. Then,

$$
\mathcal{X}(\omega) \phi=\phi \quad \text { and } \quad(\mathcal{X}(\omega) \phi)(\theta)=\phi(\theta) \quad \text { for } \quad \theta \in[-r, 0]
$$

Since

$$
(\mathcal{X}(\omega) \phi)(0)=\mathcal{T}_{0}(\omega) \phi(0)
$$

then

$$
\phi(0)=\mathcal{T}_{0}(\omega) \phi(0),
$$

and hence

$$
\phi(0) \in \operatorname{ker}\left(I-\mathcal{T}_{0}(\omega)\right) .
$$

Conversely, let $x \in \operatorname{ker}\left(I-\mathcal{T}_{0}(\omega)\right)$ and $\phi_{n}(\theta)=\mathcal{T}_{0}(n \omega+\theta) x \quad$ for $n \geq\left[\frac{r}{\omega}\right]+1$, where [.] denotes the integer part. Then,

$$
\mathcal{T}_{0}(t+\omega) x=\mathcal{T}_{0}(t) \mathcal{T}_{0}(\omega) x=\mathcal{T}_{0}(t) x \quad \text { for } \quad t \geq 0
$$

and $\phi_{n}(\theta)$ is independent of the integer $n$ and then

$$
\phi_{n}(\theta)=\mathcal{T}_{0}(\omega+\theta) x=\phi(\theta) \quad \text { and } \quad \phi(0)=x
$$

If $\omega+\theta \geq 0$, then

$$
(\mathcal{X}(\omega) \phi)(\theta)=\mathcal{T}_{0}(\omega+\theta) \phi(0)=\mathcal{T}_{0}(\omega+\theta) x=\phi(\theta) .
$$

If $\omega+\theta \leq 0$, then

$$
\begin{aligned}
(\mathcal{X}(\omega) \phi)(\theta) & =\phi(\omega+\theta)=\phi_{n}(\omega+\theta) \\
& =\mathcal{T}_{0}(\theta+\omega+n \omega) x=\mathcal{T}_{0}(n \omega+\theta) x \\
& =\phi_{n}(\theta)=\phi(\theta),
\end{aligned}
$$

hence,

$$
\mathcal{X}(\omega) \phi=\phi,
$$

which implies that $\phi \in \operatorname{ker}(I-\mathcal{X}(\omega))$. Moreover, $\operatorname{ker}\left(I-\mathcal{T}_{0}(\omega)\right)$ is mapped bijectively onto the space $\operatorname{ker}(I-\mathcal{X}(\omega))$. Therefore,

$$
\operatorname{dim} \operatorname{ker}(I-\mathcal{X}(\omega))=\operatorname{dim} \operatorname{ker}\left(I-\mathcal{T}_{0}(\omega)\right) .
$$

Let us define the constant $m_{\omega}$ by

$$
m_{\omega}=\sup _{0 \leq t \leq \omega}\left|\mathcal{T}_{0}(t)\right|
$$

As it is shown in [22], the proof is omitted here, if $I-\mathcal{T}_{0}(\omega)$ is semi-Fredholm on $\overline{D(\mathcal{A})}$, then, the operator defined by

$$
S_{M}:=I-\mathcal{T}_{0}(\omega): M \rightarrow \operatorname{Im}\left(I-\mathcal{T}_{0}(\omega)\right),
$$


is bijective, such that $M$ is a subset of $\mathbb{E}$ and $\overline{D(\mathcal{A})}$ is decomposed as

$$
\overline{D(\mathcal{A})}=\operatorname{ker}\left(I-\mathcal{T}_{0}(\omega)\right) \oplus M .
$$

Let $S_{M}^{-1}$ be the inverse operator of $S_{M}$ and let $k \in \mathbb{N}^{*}$ such that

$$
(k-1) \omega<r \leq k \omega .
$$

We set

$$
I_{k}=[-r,-(k-1) \omega) \text { and } I_{p}=[-p \omega,-(p-1) \omega) \quad \text { for } \quad p=1,2, \ldots, k-1 \quad \text { with } \quad k \geq 2 .
$$

Let $\mathcal{G}$ be a linear operator defined from $D(\mathcal{G})$ to $\mathcal{C}_{0}$ by

$$
(\mathcal{G} \phi)(\theta)=\sum_{j=0}^{p-1} \phi(\theta+j \omega)+\mathcal{T}_{0}(\theta+p \omega) S_{M}^{-1} \phi(0) \quad \text { for } \quad \theta \in I_{p},
$$

with

$$
D(\mathcal{G})=\left\{\phi \in \mathcal{C}_{0}: \phi(0) \in \operatorname{Im}\left(I-\mathcal{T}_{0}(\omega)\right)\right\} .
$$

Clearly, for $\theta \in I_{p}, p=1,2, \ldots, k$,

$$
\|\mathcal{G} \phi\|_{\mathcal{C}}=\sup _{\theta \in[-r, 0]}|(\mathcal{G} \phi)(\theta)| \leq \sum_{j=0}^{p-1}\|\phi\|_{\mathcal{C}}+\sup _{s \in[0, \omega]}\left|\mathcal{T}_{0}(s)\right|\left|S_{M}^{-1} \| \phi(0)\right| .
$$

Then

$$
\|\mathcal{G} \phi\|_{\mathcal{C}} \leq\left(k+m_{\omega}\left|S_{M}^{-1}\right|\right)\|\phi\|_{\mathcal{C}}
$$

Consequently, we have the following Theorem.

Theorem 3.4. $I-\mathcal{T}_{0}(\omega)$ is semi-Fredholm on $\overline{D(\mathcal{A})}$ if and only if $I-\mathcal{X}(\omega)$ is semi-Fredholm on $\mathcal{C}_{0}$

To prove Theorem 3.4, we need the following Lemma

Lemma 3.5 ([14]).

$$
\operatorname{Im}(I-\mathcal{X}(\omega))=D(\mathcal{G})
$$

Proof of Theorem 3.4. Suppose that $\operatorname{Im}\left(I-\mathcal{T}_{0}(\omega)\right)$ is closed, let $\phi_{n} \in D(\mathcal{G})$ such that $\phi_{n} \rightarrow \phi$ as $n \rightarrow \infty$. Then

$$
\phi_{n}(0) \in \operatorname{Im}\left(I-\mathcal{T}_{0}(\omega)\right) \quad \text { and } \quad \phi_{n}(0) \rightarrow \phi(0) \in \operatorname{Im}\left(I-\mathcal{T}_{0}(\omega)\right),
$$

which implies that $\phi \in D(\mathcal{G})$ and $D(\mathcal{G})$ is closed. Lemma 3.5 implies that $\operatorname{Im}(I-\mathcal{X}(\omega))$ is closed. Now, we suppose that $\operatorname{Im}(I-\mathcal{X}(\omega))$ is closed and $x_{n} \in \operatorname{Im}\left(I-\mathcal{T}_{0}(\omega)\right)$ such that $x_{n} \rightarrow x$ as $n \rightarrow \infty$. Let $\phi_{n}, \phi \in \mathcal{C}_{0}$ be such that $\phi_{n}(\theta)=x_{n}$ and $\phi(\theta)=x$. It is clear that $\phi_{n} \rightarrow \phi$ as $n \rightarrow \infty$ and by Lemma 3.5 we have that $\left(\phi_{n}\right) \in \operatorname{Im}(I-\mathcal{X}(\omega))$. Then $\phi \in \operatorname{Im}(I-\mathcal{X}(\omega))$ and $\phi(0)=x \in \operatorname{Im}\left(I-\mathcal{T}_{0}(\omega)\right)$. Consequently, $\operatorname{Im}\left(I-\mathcal{T}_{0}(\omega)\right)$ is closed. Therefore, by the use of Proposition 3.3 we obtain the desired result. 
In the nondensely defined case, we can prove the following result as in [22], the proof is omitted here.

Theorem 3.6. Suppose that $I-\mathcal{T}_{0}(\omega)$ is semi-Fredholm on $\overline{D(\mathcal{A})}$ such that $\operatorname{dim} \operatorname{ker}\left(I-\mathcal{T}_{0}(\omega)\right)=n$. If

$$
|\mathcal{Z}(\omega)|<\frac{1}{2 \widetilde{c}(1+\sqrt{n})}
$$

Then,

$$
I-\mathcal{Y}(\omega) \in \Phi_{+}\left(\mathcal{C}_{0}\right) \quad \text { and } \quad \operatorname{dim} \operatorname{ker}(I-\mathcal{Y}(\omega)) \leq n
$$

Proposition 3.7. Suppose that $I-\mathcal{T}_{0}(\omega)$ is semi-Fredholm on $\overline{D(\mathcal{A})}$. If $|L|$ satisfies

$$
|L|<\frac{\log \left(\frac{e^{-\omega_{0}^{+} \omega}}{2 M_{0} \widetilde{c}(1+\sqrt{n})}+1\right)}{M_{0} \bar{M} \omega} .
$$

Then,

$$
I-\mathcal{Y}(\omega) \in \Phi_{+}\left(\mathcal{C}_{0}\right) \quad \text { and } \quad \operatorname{dim} \operatorname{ker}(I-\mathcal{Y}(\omega)) \leq n
$$

Proof. By the inequality (3.2), it follows that

$$
M_{0} e^{\omega_{0}^{+} \omega}\left(e^{M_{0} \bar{M}|L| \omega}-1\right)<\frac{1}{2 \widetilde{c}(1+\sqrt{n})},
$$

and by Proposition 3.1, one has

$$
|\mathcal{Z}(\omega)|<\frac{1}{2 \widetilde{c}(1+\sqrt{n})}
$$

Theorem 3.6 gives that

$$
I-\mathcal{Y}(\omega) \in \Phi_{+}\left(\mathcal{C}_{0}\right) \quad \text { and } \quad \operatorname{dim} \operatorname{ker}(I-\mathcal{Y}(\omega)) \leq n
$$

\section{Periodic solutions for Equation (1.1)}

To discuss the existence of periodic solutions of Equation (1.1), we introduce the following fixed point Theorem for a linear affine map $T$ defined from $\mathbb{E}$ to $\mathbb{E}$ by

$$
T u=\bar{T} u+v \quad \text { for } \quad u \in \mathbb{E},
$$

where $\bar{T} \in B(\mathbb{E})$ and $v \in \mathbb{E}$ is fixed. Let $\mathcal{F}_{T}$ be the set of all fixed points of the map $T$.

Theorem 4.1 ([5]). Let $T$ be a linear affine map on a Banach space $\mathbb{E}$ such that the range $\operatorname{Im}(I-\bar{T})$ is closed. If there is an $u_{0} \in \mathbb{E}$ such that $\left\{T^{k} u_{0}, k \in \mathbb{N}\right\}$ is bounded in $\mathbb{E}$, then $\mathcal{F}_{T} \neq \varnothing$.

If there exists some $v \in \mathcal{F}_{T}$, then

$$
\mathcal{F}_{T}=v+\operatorname{ker}(I-\bar{T}) .
$$


$\operatorname{dim} \mathcal{F}_{T}$ is defined as

$$
\operatorname{dim} \mathcal{F}_{T}=\operatorname{dim} \operatorname{ker}(I-\bar{T}) .
$$

If $I-\bar{T} \in \Phi_{+}(\mathbb{X})$. Then, Theorem 4.1 is refined as follows

Theorem $4.2([22])$. Let $T$ be a linear affine map on a Banach space $\mathbb{E}$. If $I-\bar{T} \in \Phi_{+}(\mathbb{E})$ and if there exists an $u_{0} \in \mathbb{E}$ such that $\left\{T^{k} u_{0}, k \in \mathbb{N}\right\}$ is bounded, then $\mathcal{F}_{T} \neq \varnothing$ and $\operatorname{dim} \mathcal{F}_{T}$ is finite.

Through the rest of this work, we suppose that

$\left(\mathbf{H}_{\mathbf{1}}\right) h$ is an $\omega$-periodic function.

Furthermore, by property $(\mathcal{R})$ we mean the following equivalence:

Equation (1.1) has an $\omega$-periodic solution if and only if it has a bounded one on the positive real line. Then, we have the following result.

Theorem 4.3. Under assumptions $\left(\mathbf{H}_{\mathbf{0}}\right)$ and $\left(\mathbf{H}_{\mathbf{1}}\right)$. If $I-\mathcal{T}_{0}(\omega)$ is semi-Fredholm on $\overline{D(\mathcal{A})}$ and if the operator $L$ satisfies the following estimate

$$
|L|<\frac{\log \left(\frac{e^{-\omega_{0}^{+} \omega}}{2 M_{0} \widetilde{c}(1+\sqrt{n})}+1\right)}{M_{0} \bar{M} \omega},
$$

where $\widetilde{c}$ and $n$ are the constants given in Theorem 3.6. Then, Equation (1.1) satisfies the property $(\mathcal{R})$.

Proof. Let $y(., \psi, h)$ be the solution of Equation (1.1). We introduce the Poincare map $\mathcal{P}$ defined from $\mathcal{C}_{0}$ to $\mathcal{C}_{0}$ as follows

$$
\mathcal{P}_{\omega}(\psi)=y_{\omega}(., \psi, h)
$$

Then,

$$
\mathcal{P}_{\omega} \psi=y_{\omega}(., \psi, 0)+y_{\omega}(., 0, h)
$$

and hence $\mathcal{P}_{\omega}$ is an affine map such that

$$
\mathcal{P}_{\omega} \psi=\bar{P} \psi+\varphi
$$

with

$$
\bar{P} \psi=y_{\omega}(., \psi, 0) \quad \text { and } \quad \varphi=y_{\omega}(., 0, h) .
$$

According to the second section, $\bar{P}$ is decomposed as

$$
\bar{P}=\mathcal{X}(\omega)+\mathcal{Z}(\omega) .
$$

Moreover, Proposition 3.7 gives that

$$
I-\bar{P} \in \Phi_{+}\left(\mathcal{C}_{0}\right) .
$$


Now, let $y(., \psi, h)$ denote the bounded solution of Equation (1.1) on $\mathbb{R}^{+}$. Thus, for each $n \in \mathbb{N}$, we have

$$
\mathcal{P}_{\omega}^{n} \psi=y_{n \omega}(., \psi, h)
$$

and then $\left(\mathcal{P}_{\omega}^{n} \psi\right)_{n \geq 0}$ is a bounded sequence in $\mathcal{C}_{0}$. All conditions of Theorem 4.2 are satisfied and then $\mathcal{F}_{\mathcal{P}_{\omega}} \neq \varnothing$, which yields an $\omega$-periodic solution of Equation (1.1).

Corollary 4.4. Under assumptions $\left(\mathbf{H}_{\mathbf{0}}\right)$ and $\left(\mathbf{H}_{\mathbf{1}}\right)$. If $I-\mathcal{T}_{0}(\omega)$ is semi-Fredholm in $\overline{D(\mathcal{A})}$ and if $|L|$ satisfies the following inequality

$$
|L|<\frac{\log \left(\frac{e^{-\omega_{0}^{+} \omega}}{2 M_{0}\left(k+m_{\omega}\left|S_{M}^{-1}\right|\right)(1+\sqrt{n})}+1\right)}{M_{0} \bar{M} \omega} .
$$

Then, Equation (1.1) satisfies the property $(\mathcal{R})$.

To establish the proof, we need the following Lemma.

Lemma 4.5 ([14]). Suppose that $I-\mathcal{T}_{0}(\omega)$ is semi-Fredholm on $\overline{D(\mathcal{A})}$. If there exists a constant $\widetilde{c}>0$ such that

$$
\|\mathcal{G} \psi\|_{\mathcal{C}} \leq \widetilde{c}\|\psi\|_{\mathcal{C}} \quad \text { for all } \quad \psi \in D(\mathcal{G})
$$

Then,

$$
|[\varphi]| \leq \widetilde{c}\|(I-\mathcal{X}(\omega)) \varphi\|_{\mathcal{C}} \quad \text { for all } \quad \varphi \in \mathcal{C}_{0}
$$

Proof of Corollary 4.4: Since,

$$
|L|<\frac{\log \left(\frac{e^{-\omega_{0}^{+} \omega}}{2 M_{0}\left(k+m_{\omega}\left|S_{M}^{-1}\right|\right)(1+\sqrt{n})}+1\right)}{M_{0} \bar{M} \omega} .
$$

it follows that,

$$
\left(k+m_{\omega}\left|S_{M}^{-1}\right|\right)\left(e^{M_{0} \bar{M} \omega|L|}-1\right)<\frac{e^{-\omega_{0}^{+} \omega}}{2 M_{0}(1+\sqrt{n})} .
$$

Lemma 4.5 and estimation (3.1) implies that

$$
\widetilde{c} \leq k+m_{\omega}\left|S_{M}^{-1}\right|
$$

and then

$$
\widetilde{c}\left(e^{M_{0} \bar{M} \omega|L|}-1\right)<\frac{e^{-\omega_{0}^{+} \omega}}{2 M_{0}(1+\sqrt{n})} .
$$

Finally

$$
|L|<\frac{\log \left(\frac{e^{-\omega_{0}^{+} \omega}}{2 M_{0} \widetilde{c}(1+\sqrt{n})}+1\right)}{M_{0} \bar{M} \omega} .
$$

Now, Theorem 4.3 shows that Equation (1.1) satisfies the property $(\mathcal{R})$. 
In the particular case where the semigroup $\left(\mathcal{T}_{0}(t)\right)_{t \geq 0}$ is exponentially stable, we have the following Theorem.

Theorem 4.6. Under assumptions $\left(\mathbf{H}_{\mathbf{0}}\right)$ and $\left(\mathbf{H}_{\mathbf{1}}\right)$. If the semigroup $\left(\mathcal{T}_{0}(t)\right)_{t \geq 0}$ is exponentially stable and if the operator $L$ satisfies the following inequality

$$
|L|<\frac{\log \left(\frac{1}{2 M_{0}\left(k+m_{\omega}\left|S_{M}^{-1}\right|\right)}+1\right)}{M_{0} \bar{M} \omega} .
$$

Then, Equation (1.1) satisfies the property $(\mathcal{R})$.

Proof. From the exponential stability of $\left(\mathcal{T}_{0}(t)\right)_{t \geq 0}$, we have

$$
\omega_{\text {ess }}\left(\mathcal{T}_{0}\right)=\lim _{t \rightarrow+\infty} \frac{1}{t} \log \left|\mathcal{T}_{0}(t)\right|_{\alpha} \leq \lim _{t \rightarrow+\infty} \frac{1}{t} \log \left|\mathcal{T}_{0}(t)\right|=-\omega_{0}<0 .
$$

Consequently,

$$
r_{\text {ess }}\left(\mathcal{T}_{0}(\omega)\right)=\exp \left(\omega \omega_{\text {ess }}\left(\mathcal{T}_{0}\right)\right)<1 .
$$

Which implies that $\operatorname{Im}\left(I-\mathcal{T}_{0}(\omega)\right)$ is closed. On the other hand, one has

$$
\left|\mathcal{T}_{0}(\omega)^{n}\right|=\left|\mathcal{T}_{0}(n \omega)\right| \leq M_{0} e^{-\omega_{0} n \omega}
$$

and

$$
\left|\mathcal{T}_{0}(n \omega)\right|^{\frac{1}{n}} \leq M_{0}^{\frac{1}{n}} e^{-\omega_{0} \omega},
$$

which implies that the spectral radius is estimated as

$$
r\left(\mathcal{T}_{0}(\omega)\right)=\lim _{n \rightarrow+\infty}\left|\mathcal{T}_{0}(\omega)^{n}\right|^{\frac{1}{n}} \leq e^{-\omega_{0} \omega} \lim _{n \rightarrow+\infty} M_{0}^{\frac{1}{n}}<\lim _{n \rightarrow+\infty} M_{0}^{\frac{1}{n}}<1 .
$$

Consequently

$$
1 \notin \sigma\left(\mathcal{T}_{0}(\omega)\right) \quad \text { and } \quad n=\operatorname{dim} \operatorname{ker}\left(I-\mathcal{T}_{0}(\omega)\right)=0 .
$$

All conditions of Corollary 4.4 are satisfied with $n=0$. Then, Equation (1.1) satisfies the property $(\mathcal{R})$.

\section{Application}

In order to apply our theoretical results, we consider the following delayed partial differential equation:

$$
\left\{\begin{aligned}
\frac{\partial}{\partial t} y(t, \zeta) & =\frac{\partial^{2}}{\partial \zeta^{2}} y(t, \zeta)-a y(t, \zeta)+b y(t-r, \zeta)+g(t, \zeta) & & \text { for } t \in \mathbb{R}^{+} \text {and } \zeta \in \mathbb{R} \\
y(\theta, \zeta) & =\psi_{0}(\theta, \zeta) & & \text { for } \theta \in[-r, 0] \text { and } \zeta \in \mathbb{R}
\end{aligned}\right.
$$

where $a$ and $b$ are positive constants, $g: \mathbb{R} \times \mathbb{R} \rightarrow \mathbb{R}$ and $\phi:[-r, 0] \times \mathbb{R} \rightarrow \mathbb{R}$ are continuous functions where $\phi(\theta, \zeta)$ has a finite limit at $\pm \infty$. 
Note that Equation (5.1) can be written in the form of Equation (1.1). In fact: we set $\overline{\mathbb{R}}:=$ $[-\infty,+\infty]$ and we say that $z \in \mathbf{C}^{k}(\overline{\mathbb{R}})$ if $z \in \mathbf{C}^{k}(\mathbb{R})$ and all derivatives of $z$ up to the order $k$ have finite limits at $\pm \infty$. Then, the space of continuous functions on $\overline{\mathbb{R}}$, denoted by $\mathbb{E}=\mathbf{C}(\overline{\mathbb{R}})$, endowed with the norm

$$
\|z\|_{\infty}=\sup _{-\infty<\zeta<+\infty}|z(\zeta)|
$$

becomes a Banach space. we consider the linear operator $\Delta$ defined from $D(\Delta) \subset \mathbb{E}$ to $\mathbb{E}$ by

$$
\left\{\begin{aligned}
D(\Delta) & =\left\{z \in \mathbf{C}^{2}(\overline{\mathbb{R}}): \lim _{\zeta \rightarrow \pm \infty} z(\zeta)=0\right\} \\
\Delta z & =z^{\prime \prime} .
\end{aligned}\right.
$$

Then, we have

Lemma 5.1 ([7]).

$$
(0,+\infty) \subset \rho(\Delta)
$$

and for each $\lambda>0$

$$
\left|(\lambda I-\Delta)^{-1}\right| \leq \frac{1}{\lambda}
$$

Clearly

$$
\overline{D(\Delta)}=\left\{z \in \mathbf{C}(\overline{\mathbb{R}}): \lim _{\zeta \rightarrow \pm \infty} z(\zeta)=0\right\} .
$$

We write the part $\Delta_{0}$ of $\Delta$ in $\overline{D(\Delta)}$ as

$$
\left\{\begin{aligned}
D\left(\Delta_{0}\right) & =\left\{z \in \mathbf{C}^{2}(\overline{\mathbb{R}}): \lim _{\zeta \rightarrow \pm \infty} z(\zeta)=\lim _{\zeta \rightarrow \pm \infty} z^{\prime \prime}(\zeta)=0\right\} \\
\Delta_{0} z & =z^{\prime \prime}
\end{aligned}\right.
$$

Lemma $5.2([7]) . \Delta_{0}$ is the infinitesimal generator of a strongly continuous semigroup $\left(\mathcal{T}_{\Delta_{0}}(t)\right)_{t \geq 0}$ on $\overline{D(\Delta)}$. Furthermore,

$$
\left|\mathcal{T}_{\Delta_{0}}(t)\right| \leq 1 \quad \text { for } \quad t \geq 0
$$

Let $\mathcal{A}: D(\mathcal{A}) \subset \mathbb{E} \rightarrow \mathbb{E}$ defined by:

$$
\left\{\begin{array}{l}
D(\mathcal{A})=\left\{z \in \mathbf{C}^{2}(\overline{\mathbb{R}}): \lim _{\zeta \rightarrow \pm \infty} z(\zeta)=0\right\}, \\
\mathcal{A} z=z^{\prime \prime}-a z .
\end{array}\right.
$$

By Lemma 5.1, it is clear that

Lemma 5.3.

$$
(-a,+\infty) \subset \rho(\mathcal{A})
$$

and for each $\lambda>-a$

$$
\left|(\lambda I-\mathcal{A})^{-1}\right| \leq \frac{1}{\lambda+a} .
$$


Lemma 5.3 guarantees that the assumption $\left(\mathbf{H}_{0}\right)$ is satisfied with $\widehat{\omega}=-a$ and $\bar{M}=1$. Moreover,

$$
\overline{D(\mathcal{A})}=\left\{z \in \mathbf{C}(\overline{\mathbb{R}}): \lim _{\zeta \rightarrow \pm \infty} z(\zeta)=0\right\} \varsubsetneqq \mathbb{E} .
$$

Moreover, we write the part $\mathcal{A}_{0}$ of the linear operator $\mathcal{A}$ in $\overline{D(\mathcal{A})}$ as:

$$
\left\{\begin{array}{l}
D\left(\mathcal{A}_{0}\right)=\left\{z \in \mathbf{C}^{2}(\overline{\mathbb{R}}): \lim _{\zeta \rightarrow \pm \infty} z(\zeta)=0=\lim _{\zeta \rightarrow \pm \infty} z^{\prime \prime}(\zeta)=0\right\} \\
\mathcal{A}_{0} z=z^{\prime \prime}-a z .
\end{array}\right.
$$

Lemma 5.4. $\mathcal{A}_{0}$ is the infinitesimal generator of an exponentially stable continuous semigroup $\left(\mathcal{T}_{0}(t)\right)_{t \geq 0}$ on $\overline{D(\mathcal{A})}$. Moreover, for $t \geq 0$, we have

$$
\left|\mathcal{T}_{0}(t)\right| \leq e^{-a t}
$$

Consider the following notations:

$$
\left\{\begin{array}{c}
y(t)(\zeta)=y(t, \zeta) \quad \text { for } \quad t \in \mathbb{R}^{+}, \quad \zeta \in \mathbb{R} \\
\psi(\theta)(\zeta)=\psi_{0}(\theta, \zeta) \quad \text { for } \quad \theta \in[-r, 0], \quad \zeta \in \mathbb{R}
\end{array}\right.
$$

and define the function $L: \mathcal{C} \rightarrow \mathbb{E}$ as follows

$$
L(\phi)(\zeta)=b \phi(-r)(\zeta) \quad \text { for } \quad \zeta \in \mathbb{R} \quad \text { and } \quad \phi \in \mathcal{C}
$$

$h: \mathbb{R} \longrightarrow \mathbb{E}$ is defined by

$$
h(t)(\zeta)=g(t, \zeta) \text { for } \quad t \in \mathbb{R} \text { and } \quad \zeta \in \mathbb{R}
$$

Clearly, $L$ is a linear bounded operator from $\mathcal{C}$ to $\mathbb{E}$. Then, Equation (5.1) can be written in $\mathbb{E}$ as follows

$$
\left\{\begin{aligned}
\frac{d}{d t} y(t) & =\mathcal{A} y(t)+L\left(y_{t}\right)+h(t) \quad \text { for } \quad t \geq 0 \\
y_{0} & =\psi \in \mathcal{C} .
\end{aligned}\right.
$$

We suppose that $\lim _{\zeta \rightarrow \pm \infty} \psi_{0}(0, \zeta)=0$, then Equation (5.2) has a unique integral solution $y$ on $[-r,+\infty)$.

To get the periodicity of solutions of Equation (5.2), we suppose that

$\left(\mathbf{H}_{\mathbf{2}}\right) b<a$.

Let $\rho=1+\frac{|h|_{\infty}}{a-b}$ where $|h|_{\infty}=\sup _{0 \leq t \leq \omega}|h(t)|$. Then, we have

Lemma 5.5. Under assumption $\left(\mathbf{H}_{\mathbf{2}}\right)$. For every $\psi \in \mathcal{C}$ such that $\|\psi\|_{\mathcal{C}}<\rho$, the solution of Equation (5.2) is bounded by $\rho$ on $\mathbb{R}^{+}$. 
Proof. We proceed by contradiction. Let

$$
t_{*}=\inf \{t>0:|y(t, \psi)|>\rho\} .
$$

From the continuity of $y$, one has

$$
\left|y\left(t_{*}, \psi\right)\right|=\rho,
$$

and there is $\alpha>0$, with

$$
|y(t, \psi)|>\rho \text { for each } t \in\left(t_{*}, t_{*}+\alpha\right) .
$$

Applying the variation-of-constants formula for Equation (5.2) with the initial value $\psi$,

$$
y(t)=\mathcal{T}_{0}(t) \psi(0)+\lim _{\lambda \rightarrow+\infty} \lambda \int_{0}^{t} \mathcal{T}_{0}(t-s) R(\lambda, \mathcal{A})\left(L\left(y_{s}\right)+h(s)\right) d s .
$$

Then, for $t \geq 0$

$$
\left|y\left(t_{*}, \psi\right)\right| \leq\left|\mathcal{T}_{0}\left(t_{*}\right)\right||\psi(0)|+\int_{0}^{t_{*}}\left|\mathcal{T}_{0}\left(t_{*}-s\right)\right|\left(\left|L\left(y_{s}\right)\right|+|h(s)|\right) d s .
$$

Since for $0<s<t_{*}$, it follows that $-r \leq s-r \leq t_{*}-r<t_{*}$ and then

$$
\left|L\left(y_{s}\right)\right|=b|y(s-r)| \leq b \rho,
$$

hence

$$
\begin{aligned}
\left|y\left(t_{*}, \psi\right)\right| & \leq \rho e^{-a t_{*}}+\left(b \rho+|h|_{\infty}\right) \int_{0}^{t_{*}} e^{-a\left(t_{*}-s\right)} d s \\
& \leq \rho e^{-a t_{*}}+\frac{\left(1-e^{-a t_{*}}\right)}{a}\left(b \rho+|h|_{\infty}\right) .
\end{aligned}
$$

Consequently,

$$
\begin{aligned}
\left|y\left(t_{*}, \psi\right)\right| & \leq \rho e^{-a t_{*}}+(b \rho+(a-b)(\rho-1)) \frac{\left(1-e^{-a t_{*}}\right)}{a} \\
& \leq \rho e^{-a t_{*}}+\left(\rho-1+\frac{b}{a}\right)\left(1-e^{-a t_{*}}\right) \\
& \leq \rho e^{-a t_{*}}+\rho\left(1-e^{-a t_{*}}\right) \\
& \leq \rho
\end{aligned}
$$

which contradicts the definition of $t_{*}$, and we deduce that

$$
|y(t, \psi)| \leq \rho \quad \text { for } \quad t \geq 0
$$

To discuss the periodicity of solutions of Equation (5.2), we assume that:

$\left(\mathbf{H}_{\mathbf{3}}\right) h$ is an $\omega$-periodic function in $t$.

Theorem 5.6. Suppose that $\left(\mathbf{H}_{\mathbf{2}}\right)$ and $\left(\mathbf{H}_{\mathbf{3}}\right)$ hold true. If

$$
|L|<\omega^{-1} \log \left(\frac{\left(1-e^{-a \omega}\right)(1+2 k)+2}{2+2 k\left(1-e^{-a \omega}\right)}\right),
$$

then, Equation (5.2) has an w-periodic solution. 
Proof. Let $m_{\omega}$ be the constant defined by

$$
m_{\omega}=\sup _{0 \leq t \leq \omega}\left|\mathcal{T}_{0}(t)\right|
$$

Then

$$
m_{\omega} \leq \sup _{0 \leq t \leq \omega} e^{-a t}=1
$$

Moreover, since $\left|\mathcal{T}_{0}(\omega)\right|<1$, one has

$$
\begin{aligned}
\left|S_{M}^{-1}\right|=\left|\left(I-\mathcal{T}_{0}(\omega)\right)^{-1}\right| & \leq \frac{1}{I-\left|\mathcal{T}_{0}(\omega)\right|} \\
& \leq \frac{1}{1-e^{-a \omega}} .
\end{aligned}
$$

Thus,

$$
k+m_{\omega}\left|S_{M}^{-1}\right| \leq k+\frac{1}{1-e^{-a \omega}}
$$

and

$$
\begin{aligned}
|L| & <\omega^{-1} \log \left(\frac{\left(1-e^{-a \omega}\right)(1+2 k)+2}{2+2 k\left(1-e^{-a \omega}\right)}\right) \\
& <\omega^{-1} \log \left(\frac{1}{2\left(k+m_{\omega}\left|S_{M}^{-1}\right|\right)}+1\right) .
\end{aligned}
$$

All condition of Theorem 4.6 are satisfied. Then, Lemma 5.5 implies that Equation (5.2) has an $\omega$-periodic solution.

\section{Acknowledgement}

The authors would like to thank the anonymous referees for their constructive comments and valuable suggestions, which are helpful to improve the quality of this paper. 


\section{References}

[1] M. Adimy and K. Ezzinbi, "Local existence and linearized stability for partial functional differential equations", Dyn. Syst. Appl., vol. 7, no. 3, pp. 389-404, 1998.

[2] W. Arendt, C. J. K. Batty, M. Hieber and F. Neubrander, Vector valued Laplace transforms and Cauchy problems, Monographs in Mathematics, vol. 96, Basel: Birkhäuser Verlag, 2001.

[3] W. Arendt, A. Grabosch, G. Greiner, U. Groh, H. P. Lotz, U. Moustakas, R. Nagel, B. Neubrander and U. Schlotterbeck, One-parameter semigroup of positive operators, Lecture Notes in Mathematics, vol. 1184, Berlin: Springer-Verlag, 1984.

[4] T. Burton, Stability and periodic solutions of ordinary differential equation and functional differential equations, New York: Academic Press, 1985.

[5] S. N. Chow and J. K. Hale, "Strongly limit-compact maps", Funkcial. Ekvac., vol. 7, pp. 31-38, 1974.

[6] G. Da Prato and E. Sinestrari, "Differential operators with nondense domains", Ann. Scuola Norm. Sup. Pisa Cl. Sci. (4), vol. 14, no. 2, pp. 285-344, 1987.

[7] N. Dunford and J. T. Schwartz, Linear operators, Part I, New York: John Wiley \& Sons Inc, 1987.

[8] A. Elazzouzi and K. Ezzinbi, "Ultimate boundedness and periodicity for some partial functional differential equations with infinite delay", J. Math. Anal. Appl., vol. 329, no. 1, pp. 498-514, 2007.

[9] K. Ezzinbi, "A survey on new methods for partial functional differential equations and applications", Afr. Mat., vol. 31, no. 1, pp. 89-113, 2020.

[10] K. Ezzinbi and M. Taoudi, "Periodic solutions and attractiveness for some partial functional differential equations with lack of compactness", Proc. Amer. Math. Soc., vol. 149, no. 3, pp. 1165-1174, 2021.

[11] K. J. Engel and R. Nagel, One-parameter semigroups for linear evolution equations, Graduate Texts in Mathematics, vol. 194, New York: Springer-Verlag, 2000.

[12] J. Hale and O. Lopes, "Fixed point theorems and dissipative processes", J. Differential Equations, vol. 13, no. 2, pp. 391-402, 1973.

[13] H. R. Henríquez, "Periodic solutions of quasi-linear partial functional differential equations with unbounded delay", Funkcial. Ekvac., vol. 37, no. 2, pp. 329-343, 1994. 
[14] Y. Hino, T. Naito, N. Van Minh and J. Son Shin, Almost periodic solutions of differential equations in Banach spaces, Stability and Control: Theory, Methods and Applications, vol. 15, London: Taylor \& Francis, 2002.

[15] Y. Hino, S. Murakami and T. Yoshizawa, "Existence of almost periodic solutions of some functional differential equations in a Banach space", Tohoku Math. J., vol. 49, no. 1, pp. 133-147, 1997.

[16] G. M. N'Guérékata, Hui-Sheng Ding and W. Long, "Existence of pseudo almost periodic solutions for a class of partial functional differential equations", Electron. J. Differential Equations, vol. 2013, no. 104, 14 pages, 2013.

[17] M. Kostić, Almost periodic and almost automorphic type solutions to integro-differential equations, Berlin: W. de Gruyter, 2019.

[18] B. M. Levitan, Almost periodic functions (in Russian), Moscow: Gosudarstv. Izdat. Tehn.Teor. Lit., 1953.

[19] R. D. Nussbaum, "The radius of essential spectrum", Duke Math. J., vol. 37, pp. 473-478, 1970.

[20] A. Pazy, Semigroups of linear operators and applications to partial differential equations, Applied Mathematical Sciences, vol. 44, New York: Springer-Verlag, 1983.

[21] M. Schechter, Principles of functional analysis, New York: Academic Press, 1971.

[22] J. S. Shin and T. Naito, "Semi-Fredholm operators and periodic solution for linear functionaldifferential equations", J. Differential Equations, vol. 153, no. 2, pp. 407-441, 1999.

[23] C. C. Travis and G. F. Webb, "Existence and stability for partial functional differential equations", Trans. Amer. Math. Soc., vol. 200, pp. 395-418, 1974.

[24] J. Massera, "The existence of periodic solutions of differential equations", Duke Math. J., vol. 17, pp. 457-475, 1950.

[25] J. Wu, Theory and applications of partial functional differential equations, Applied Mathematical Sciences, vol. 119, New York: Springer-Verlag, 1996.

[26] T. Yoshizawa, Stability theory by Liapunov's second method, Publications of the Mathematical Society of Japan, vol. 9, Tokyo: Math. Soc. Japan, 1966. 